\title{
101. Ein Hauptidealsatz relativ-Galoisscher Zahlkörper und ein Satz über den Normenrest.
}

\author{
Von Tadao TanNaka. \\ Mathematisches Institut, Tohoku Kaiserlichen Universität, Sendai. \\ (Comm. by M. FuJiwara, M.I.A., Oct. 12, 1933.)
}

Der allgameine Hauptidealsatz:

Im Strahlklassenkörper $K$ mod. $f$ fallen alle (zu den Führer von $K / k$ primen) Ideale im Grundkörper $k$ in den Strahl mod. $\mathfrak{F}(K / k)$ wurde zuerst von Herrn Iyanaga, als eine Verallgemeinerung des Furtwänglerschen Hauptidealsatzes, bewiesen, und nachher von J. Herbrand $^{1)}$ vereinfacht. Wenn man die Definitionen des (nicht notwendig in $k$ liegenden) Führers $\mathfrak{f}(K / k)$ und Geschlechtsmoduls $\mathfrak{F}(K / k)$ auf den beliebigen relativ-algebraischen Zahlkörper $K / k$ naturgemäss yerallgemeinert, so kann man den Iyanagaschen Hauptidealsatz folgendermassen erweitern :

Satz $A$. Es sei $K / k$ ein relativ-Galoisscher Zahlkörper und $m$ die Anzahl der Strahlklassen mod. $f(K / k)$, welche in $K$ mod. $f(K / k) \mathrm{zu}-$ geordnete Idealgruppe in $k$ enthalten sind. Alle $\mathrm{zu} f(K / k)$ primen Ideale in $k$, welche mod. $\mathrm{f}(K / k)$ zu $m$ prime Ordnung haben, fallen dann in $K$ in den Strahl mod. $\mathfrak{F}(K / k)$.

Wir können auch den folgenden Satz beweisen:

Satz $B$. Es sei $K / k$ ein beliebiger relativ-algebraischer Zahlkörper, und liege das Ideal $\mathfrak{m}=\mathfrak{M}_{0} f(K / k)$ in $k$. Dann fallen alle Strahlideale mod. $\mathfrak{M}_{0} \mathfrak{F}(K / k)$ in $K$ bei der Normbildung in den Strahl mod. $\mathfrak{m}$.

Die genaue Werte der Ideale $f(K / k)$ und $\mathfrak{F}(K / k)$ sind wie folgt bestimmt: Es sei $\bar{K}$ der durch $K$ erzeugte Galoissche Körper (mit der Gruppe $G$ ) über $k ; g$ zu $K$ gehörige Untergruppe von $G ; \bar{P}, \mathfrak{P}, \mathfrak{p}$ die entsprechenden Primideale in bez. $\bar{K}, K, k ; G_{i}(i \geqq 1)$ die $(i-1)$-te Verzweigungsgruppe von $\overline{\mathfrak{P}}$ für $\bar{K} / k$ (insbesondere ist also $G_{1}$ die Trägheitsgruppe von $\overline{\mathfrak{S}}$ ). Die $\overline{\mathfrak{P}}$-Beitrag der betreffenden Ideale sind dann durch die Formeln

$$
\text { Exp. } \mathrm{f}_{\bar{P}}(K / k)=\sum_{G i \in g} \text { Ordn. } G_{i}
$$

1) J. Herbrand: Sur les théorèmes du genre principal et des idéaux principaux. Abh. Math. Sem. Hamburg, 9 (1932). 
und

$$
\text { Exp. } \mathfrak{F}_{\bar{\beta}}(K / k)=\sum_{G_{i} \in g} \operatorname{Ordn} .\left[G_{i}, g\right]
$$

gegeben.

Diese Sätze sind, bis auf einige kleinen Utberlegungen, durch Herbrandsche Methode beweisbar. 